\title{
Self mutilation with bilateral rhegmatogenous retinal detachment in tourette's syndorme treated with pars plana vitrectomy
}

\begin{abstract}
Gilles de la Tourette's syndrome, is a condition characterized by multiple motor and vocal tics, which appear in childhood and are often accompanied by behavioral symptoms. There are isolated reports which associate this syndrome with color vision deficiencies, self-induced haemophthalmous and dislocation of crystalline lens, retinal detachment and keratoconus. This is a case report of a known case of Gilles de la Tourette's Syndrome patient who presented with bilateral visual loss. The visual loss was as consequence of repeated attacks during which he punched his periorbital region with his hand. There was total rhegmatogenous retinal detachment with advanced proliferative vitreoretinopathy in both eyes. The left eye was treated with phacoemulsification vitrectomy. Retina was successfully attached and remained attached at 1 year follow up after silicone oil removal in the left eye.
\end{abstract}

Keywords: tourette's syndrome, rhegmatogenous retinal detachment, vitrectomy, case report, albania
Volume 7 Issue 4 - 2017

\author{
Nishant Taneja,' Marsida Krasniqi, ${ }^{2,3}$ Vilma \\ Mema $^{2}$ \\ 'International Eye Clinic, Albania \\ 2Department of Ophthalmology, Mother Theresa Hospital, \\ Albania \\ ${ }^{3}$ Department of Medicine, University Aleksander Moisiu, Albania
}

\begin{abstract}
Correspondence: Marsida Krasniqi, Department of Ophthalmology, Mother Theresa Hospital,Tirana, Albania, Department of Medicine, University Aleksander Moisiu, Durres, Albania,Tel +35569263306I, Email k_marsida@yahoo.it
\end{abstract}

Received: September 03, 2017 | Published: September 19 2017

\section{Introduction}

Gilles de la Tourette's syndrome, or Tourette's syndrome (TS), is a neurodevelopmental condition characterized by multiple motor and vocal tics, which appear in childhood and are often accompanied by behavioral symptoms. ${ }^{1}$ The chronic presence of at least two motor tics and one vocal tic since childhood is recognized as the key feature of Tourette's syndrome., ${ }^{1,2}$ Motor tics (facial grimacing, arm jerking, punching, blinking, alterations in gaze, rubbing of the eyes, blepharospasm, etc.) are well known as early manifestations of $\mathrm{TS}^{3}{ }^{3}$ There are isolated reports, which associate this syndrome with color vision deficiencies, self-induced vitreous hemorrhage, and dislocation of crystalline lens, retinal detachment and keratoconus. ${ }^{4,5}$ The early ocular manifestations are easily missed, as these patients are too embarrassed to complain about the visual symptoms. A delay in diagnosis of retinal detachment is not good as the RD can progress to PVR and visual prognosis in PVR cases is poor even after multiple VR procedures. ${ }^{6-8}$ In this article, we report a case of bilateral retinal detachment in a patient with TS that resulted as a consequence of repeated punching of periorbital region by hand.

\section{Case report}

This is a case of 24 years old boy who presented to us due to rapidly progressive deterioration of vision in both eyes over a period of 3 months. His past medical history included a diagnosis of Tourette syndrome (TS) and depression since the age of 14 years. He was not compliant to his medical therapy for fast 1 year. His mother noticed that he was confined to his room for past few days and was stumbling upon the objects at home. She described his motor tics as having blepharospasm, clapping, gouging his fingers into his eyes, and punching himself in the periorbital area. She decided to seek ophthalmologist opinion when she noticed exacerbation in the tic frequency and severe periorbital ecchymosis over the right eye. During the examination, patient was well oriented, did not show any vocal or motor tics and no signs of cognitive impairment. On ocular examination, there was periorbital ecchymosis in the right eye and multiple mechanical scratch marks in the periorbital region laterally and also on face (Figure 1). The nature of the external injuries was commensurate with a tendency of motor tics described by his mother. Ocular movements were full and free. Visual acuity was no perception of light in the right eye and hand movement close to face in the left eye. Slit lamp biomicroscopy showed clear cornea in both eyes (Figure 2). Right eye examination revealed posterior synechiae with near total cataract. There was evidence of KPs, mild aqueous cell and posterior sub capsular cataract in the left eye. Even though the fundus visibility was limited by vitreous hemorrhage, a total retinal detachment with proliferative vitreoretinopathy could be seen in his both eyes (Figure $3)$. Echography revealed a closed funnel retinal detachment in both eyes (Figure 4).

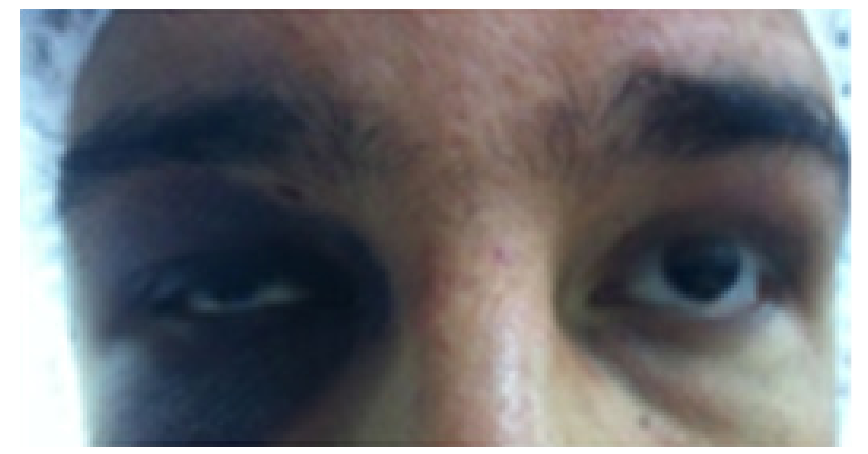

Figure I Periorbital ecchymosis in the right eye. 


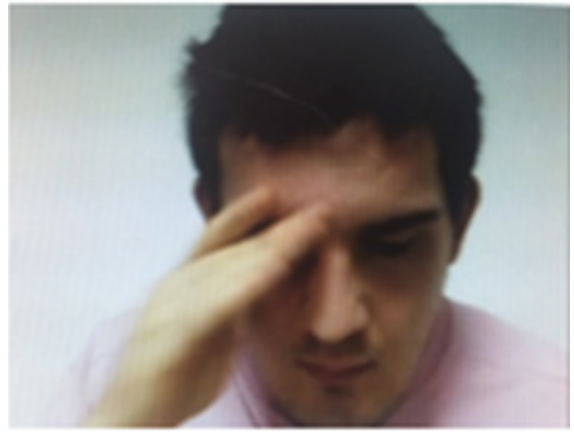

Figure 2 Mechanism of tick.

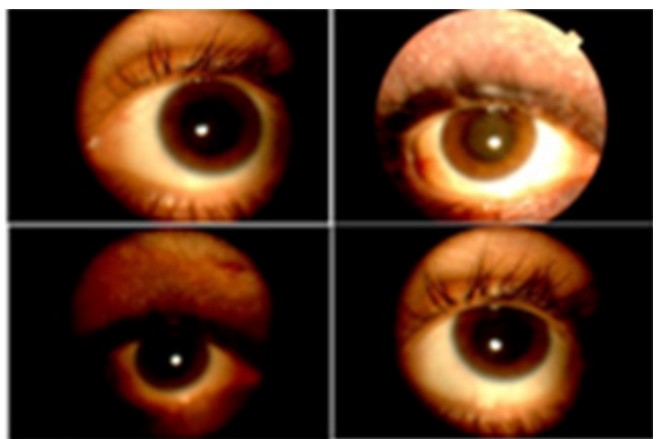

Figure 3 Anterior segments photographs.

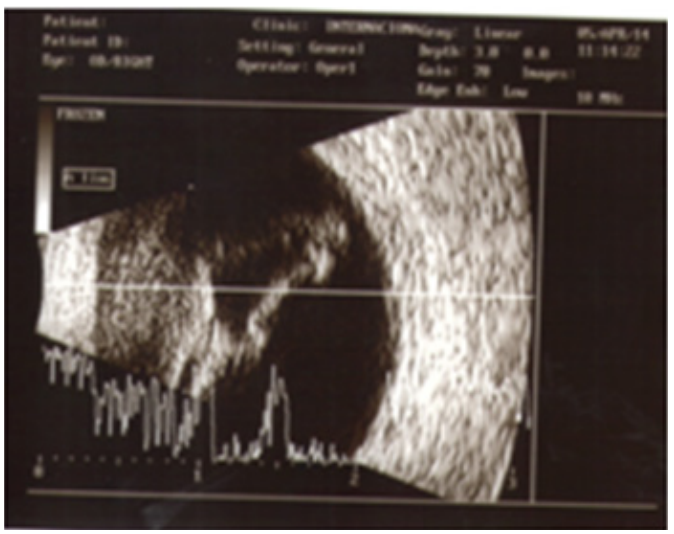

Figure 4 Echo scan from the patient.

Prognosis of vision in the right eye was nil and explained to the patient. Left eye was managed with phacoemulsification pars plana vitrectomy, endolaser and injection of silicone oil. Intraoperatively, there was inferotemporal dialysis extending from 4.00 to $7.00 \mathrm{o}$ clock position. There was extensive subretinal glial proliferation and preretinal membranes. Peripheral vitrectomy was performed with scleral depression and endolaser was performed. Because of the difficulty of maintaining a facedown position and the possibility of further self-injury, an encircling silicone belt was placed, and the eye was filled with high viscosity silicone oil. Early postoperative phase was uneventful and visual acuity improved to 20/100 with +4.00 Dsph. Patient was advised to take protective polycarbonate glasses and to patch the eye with plastic shield during sleeping. After 4 months patient presented with sudden decrease of vision in the left eye following uncontrollable punching and jabbing in the left eye. On examination, there were small bubbles of silicone oil in the anterior chamber (Figures 5\&6). Retina appeared attached (Figures 7\&8).
Patient underwent membrane peeling with silicone oil removal after psychiatric consultation and modification of medical therapy for TS. At 6 months, the retina remained attached and the visual acuity was $20 / 100$ in the left eye.

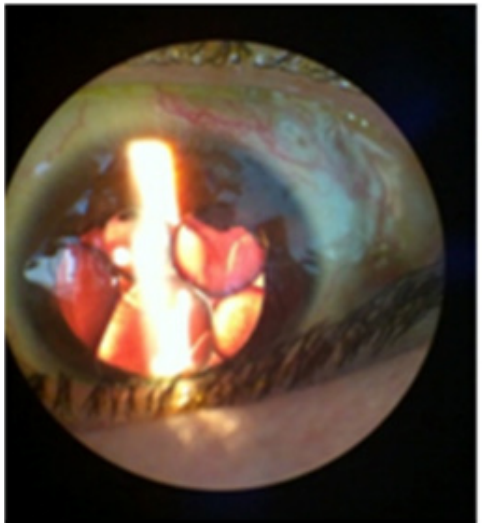

Figure 5 Bubbles of silicone oil in the anterior chamber - 4 months.

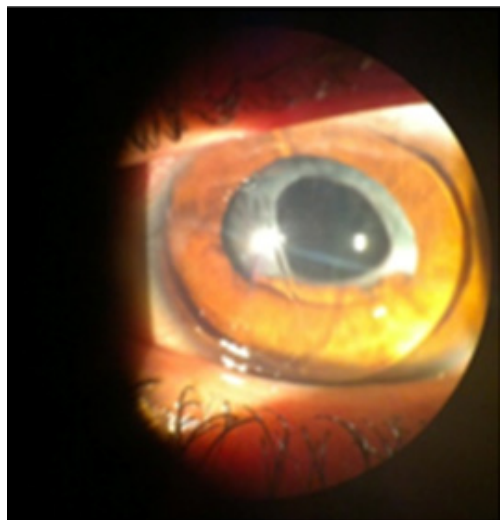

Figure 6 Bubbles of silicon oil- 5 months post operation.
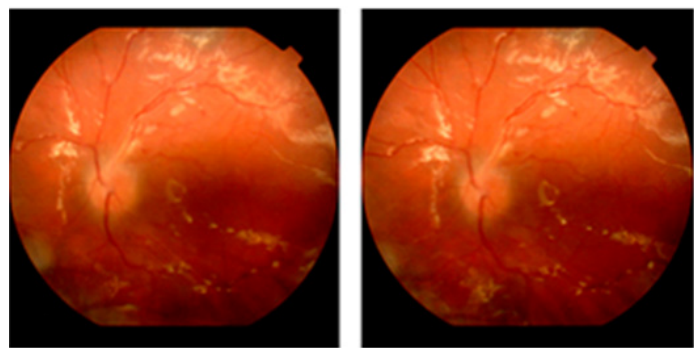

Figure 7 Attached retina with silicone oil in situ - 3 month post operative photo.
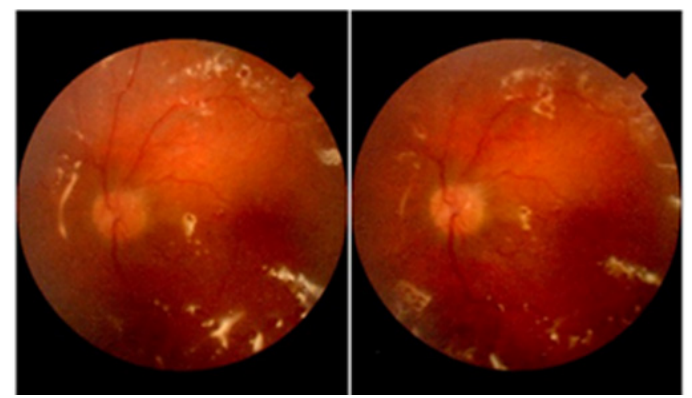

Figure 8 Attached retina with silicone oil - 6 month post operative photo. 


\section{Discussion}

Patients with TS have motor tics which are involuntary rapid and recurrent which can lead to severe ocular trauma. ${ }^{9-11}$ Tics like head banging, punching and striking of the face or periorbital region can lead to direct coup injury to eye resulting in breakdown of formed vitreous. Rapid movement of the vitreous body due to repeated self inflicted trauma caused tensile stress on the vitreous base and peripheral retina leading to formation of giant retinal tears and dialysis. ${ }^{12}$ Eye rubbing, jabbing of fingers into the eye contribute to axial myopia that may increase the traction at vitreous base in presence of partial posterior vitreous detachment. Even in our case repeated self-trauma caused retinal detachment. This syndrome is not associated with intellectual disability but owing to the potentially disabling nature of the physical symptoms and the social stigmata associated with the disorder some patients are very hideous regarding their complains. This leads to delay in the diagnosis and associated problem related to retinal detachment complicated with advanced PVR. The surgical procedures were chosen in our case to reduce the likelihood of additional retinal tears or recurrent RD resulting from continued trauma. Encircling silicone belt reduces the dynamic traction on the vitreous base and counteracts the circumferential traction and retinal foreshortening associated with advanced PVR. Vitrectomy with adequate membrane dissection directly relieves the traction on the torn retina. High viscosity silicone oil provides sufficient long-term internal tamponade. In our patient, despite the best efforts of the parents, health professionals and modification of medical therapy, self injurious behavior could not be eliminated completely which resulted in the anterior migration of the silicone oil 3 months after surgery.

\section{Conclusion}

TS can lead to bilateral rhegmatogenous RD associated with compulsive striking of the periorbital region with hand. In our case a bilateral rhegmatogenous retinal detachment was seen. We report this patient whose retina was successfully attached after 2 vitreoretinal procedures. It is important for the psychiatrist to recognize patients with TS with this kind of behavior. To avoid further self injury, these patients must use eye protection in the form of polycarbonate glasses. This case report emphasizes the importance of a multi disciplinary approach in the management of these potentially high-risk patients to avoid sight threatening eye injuries.

\section{Consent}

Written informed consent was obtained from the patient to publication of this case report.

\section{Acknowledgments}

None.

\section{Conflicts of interest}

There are no conflicts of interest.

\section{Funding}

None.

\section{References}

1. Banaschewski T, Woerner W, Rothenberger A. Premonitory sensory phenomena and suppressibility of tics in Tourette syndrome: developmental aspects in children and adolescents. Dev Med Child Neurol. 2003;45(10):700-703.

2. Conelea CA, Woods DW. Examining the impact of distraction on tic suppression in children and adolescents with Tourette syndrome. Behav Res Ther. 2008;46(11):1193-2000.

3. Leckman JF, Zhang H, Vitale A, et al. Course of tic severity in Tourette syndrome: the first two decades. Pediatrics. 1998;102(1 Pt 1):14-19.

4. Chee KY, Sachdev P. A controlled study of sensory tics in Gilles de 1a Tourette syndrome and obsessive-compulsive disorder using a structured interview. J Neurol Neurosurg Psychiatry. 1997;62(2):188-192.

5. Scharf JM, Miller LL, Gauvin CA, et al. Population prevalence of Tourette syndrome: A systematic review and meta-analysis. Mov Disord. 2015;30(2):221-228.

6. Burd L, Kerbeshian PJ, Barth A, et al. Long-term follow-up of an epidemiologically defined cohort of patients with Tourette syndrome. $J$ Child Neurol. 2001;16(6):431-437.

7. Kwak C, Vuong KD, Jankovic J. Premonitory sensory phenomenon in Tourette's syndrome. Mov Disord. 2003;18(12):1530-1533.

8. Prado HS, Rosário MC, Lee J, et al. Sensory phenomena in obsessivecompulsive disorder and tic disorders: a review of the literature. CNS Spectr. 2008;13(5):425-432.

9. Eddy CM, Rizzo R, Gulisano M, et al. Quality of life in young people with Tourette syndrome: a controlled study. J Neurol. 2010;258(2):291301 .

10. Golden G. Tourette syndrome: recent advances. Pediatr Neurol. 1986;2(4):189-192.

11. Lim S, Rezai KA, Abrams GW, et al. Self-induced, bilateral retinal detachment in Tourette syndrome. Arch Ophthalmol. 2004;122(6):930 931.

12. Kandarakis A, Karampelas M, Soumplis V, et al. A case of bilateral selfinduced keratoconus in a patient with tourette syndrome associated with compulsive eye rubbing: case report. BMC Ophthalmology. 2011;11:28. 